\title{
ON THE VARIANCE OF THE NUMBER OF REAL ROOTS OF A RANDOM TRIGONOMETRIC POLYNOMIAL*
}

\author{
K. Farahmand \\ Department of Mathematical Statistics \\ University of Cape Town \\ Rondebosch 7700, South Africa
}

\begin{abstract}
This paper provides an upper estimate for the variance of the number of real zeros of the random trigonometric polynomial $\mathrm{g}_{1} \cos \theta+\mathrm{g}_{2} \cos 2 \theta+\ldots$. $g_{n} \cos n \theta$. The coefficients $g_{i}(i=1,2, \ldots, n)$ are assumed independent and normally distributed with mean zero and variance one.
\end{abstract}

Key words: random trigonometric polynomial, number of real roots, variance.

AMS subject classification: $60 \mathrm{H}, 42$.

\section{INTRODUCTION}

Let

$$
T(\theta) \equiv T_{n}(\theta, \omega)=\sum_{i=1}^{n} g_{i}(\omega) \cos i \theta,
$$

where $g_{1}(\omega), g_{2}(\omega), \ldots, g_{n}(\omega)$ is a sequence of independent random variables defined on a probability space $(\Omega, \mathrm{A}, \mathrm{P})$ each normally distributed with mathematical expectation zero and variance one. Denote by $N(\alpha, \beta)$ the number of real roots of the

\footnotetext{
* Received: July 1989; Revised: April 1990
} 
equation $T(\theta)=0$ in the interval $(\alpha, \beta)$, where multiple roots are counted only once. Dunnage [3] showed that except for a set of functions of $T(\theta)$ of measure not larger than $(\log n)^{-1}$

$$
N(0,2 \pi)=2 n / \sqrt{3}+O\left\{n^{11 / 13}(\log n)^{3 / 13}\right\} .
$$

Later Sambandham and Renganathan [9] and Farahmand [4] generalized this result to the case where the coefficients $g_{i}$ have a non zero mean. They show that for $n$ sufficiently large the mathematical expectation of the number of real roots, EN, satisfies

$$
\mathrm{EN}(0,2 \pi) \sim(2 / \sqrt{3}) \mathrm{n} .
$$

The results for the dependent coefficients with constant correlation coefficient or otherwise are due to Renganathan and Sambandham [6] and Sambandham [7] and [8]. A comprehensive treatment of the zeros of random polynomial constitutes the greater part of a book by Bharucha-Reid and Sambandham [1] which gives a rigorous and interesting survey of earlier works in this field.

Qualls [5] resolved the only known variance of the number of real roots of a random trigonometric polynomial. Indeed he considered a different type of random polynomial,

$$
\sum_{i=0}^{n}\left(a_{i} \cos i \theta+b_{i} \sin i \theta\right)
$$

which has the property of being stationary and for which a special theorem has been developed by Cramer and Leadbetter [2]. Here we shall prove the following theorem:

Theorem. Let $g_{1}(\omega), g_{2}(\omega), \ldots, g_{n}(\omega)$ be the independent random variables 
corresponding to a Gaussian distribution with mean zero. Then the variance of the number of real roots of $T(\theta)$ satisfies

$$
\operatorname{Var} N(0,2 \pi)=O\left[n^{24 / 13}(\log n)^{16 / 13}\right]
$$

\section{OVERVIEW OF PROOF OF THE THEOREM AND SOME LEMMAS}

In general we make use of a delicate analysis suggested by the work of Dunnage in [3] with which we assume the reader is familiar. We divide the interval $(0,2 \pi)$ into intervals $I_{1}, I_{2}, \ldots, I_{s}$, each of equal length $\delta$. Then with each $I_{j}(j=1,2, \ldots, s)$, we associate the following two functions:

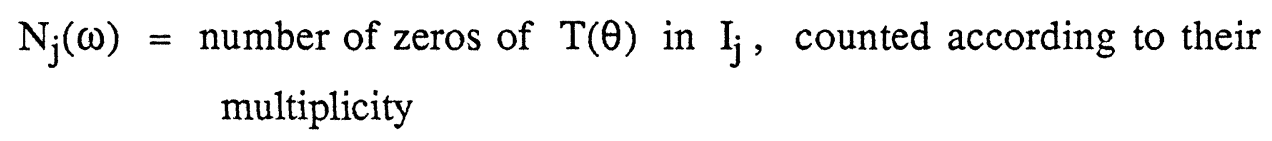

and

$$
N_{j}^{*}(\omega)= \begin{cases}N_{j}(\omega) & \text { if } N_{j}(\omega) \geq 2, \\ 0 & \text { otherwise. }\end{cases}
$$

Now if $\mathrm{T}(\mathrm{a}) \mathrm{T}(\mathrm{b}) \leq 0$ we shall say, being prompted by a graphical idea, that $\mathrm{T}(\theta)$ has a single crossover (s.c.o.) in $(a, b)$, and let

$$
\mu_{j}(\omega)= \begin{cases}1 & \text { if } T(\theta) \text { has a (s.c.o.) in } I_{j} \\ 0 & \text { otherwise }\end{cases}
$$


clearly

$$
\text { (2.1) } 0 \leq \mathrm{N}_{\mathrm{j}}(\omega)-\mu_{\mathrm{j}}(\omega) \leq \mathrm{N}_{\mathrm{j}}^{*}(\omega)
$$

For the proof of the theorem we need the following lemmas.

Lemma 1. Provided that the interval of $I$, of length $\delta=0(1 / \mathrm{n})$ does not overlap the $\varepsilon$ - neighborhood of $0, \pi$ and $2 \pi$, where $\varepsilon \sim n^{-6 / 13}(\log n)^{-4 / 13}$, the probability that $T(\theta)$ has at least two zeros (counted according to their multiplicity) in $I$ is $\mathrm{O}\left(\mathrm{n}^{3}, \delta^{3}\right)$.

Proof. This is lemma 11 of [3].

We denote by $N(\omega)$ the number of real zeros that $\mathrm{T}(\theta)$ has in $I$ and we define

$$
N^{*}(\omega)= \begin{cases}N(\omega) & \text { if } N(\omega) \geq 2 \\ 0 & \text { otherwise }\end{cases}
$$

Lemma 2. For a constant A

$$
\mathrm{E}\left[\mathrm{N}^{*}(\omega)\right]^{2}<\mathrm{A} \mathrm{n}^{3} \delta^{3} \log \mathrm{n}
$$

Proof. Suppose $\mathrm{T}(\theta)$ has at least $\mathrm{k}(\geq 2)$ zeros in $I$. Then if $I$ is divided into $2 \mathrm{p}$ equal parts where $\mathrm{p}$ is chosen as an integer satisfying $2 \mathrm{p}<\mathrm{k}<2 \mathrm{p}+1$ at least one part must contain two or more zeros, and by lemma 1 , the probability of this occurring does not exceed 


$$
\mathrm{A} 2^{\mathrm{p}} \mathrm{n}^{3}\left(\delta / 2^{\mathrm{p}}\right)^{3}=\mathrm{An}^{3} \delta^{3} 2^{-2 \mathrm{p}}<\mathrm{An}^{3} \delta^{2} / \mathrm{k}^{2} .
$$

Hence if $\mathrm{q}_{\mathrm{k}}$ is the probability that $\mathrm{T}(\theta)$ has at least $\mathrm{k}$ zeros in $I$, we have

$$
\mathrm{q}_{\mathrm{k}}<\mathrm{A} \mathrm{n}^{3} \delta^{3} / \mathrm{k}^{2} \text {. }
$$

Now we find the mathematical expectation of $\mathrm{N}^{* 2}$ as

$$
\begin{aligned}
E\left[N^{2}\right] & =\sum_{k=2}^{n} k^{2} \operatorname{Prob}(n=k) \sum_{k=2}^{n} k^{2}\left(q_{k}-q_{k+1}\right) \\
& =\sum_{k=2}^{n} k^{2} q_{k}-\sum_{k=3}^{n+1}(k-1)^{2} q_{k} \\
& \leq 4 q_{2}+\sum_{k=3}^{n+1}(2 k-1) q_{k}<\operatorname{An}^{3} \delta^{3} \log n
\end{aligned}
$$

which completes the proof of lemma 2.

Now we define

$$
\alpha_{j}=E\left(N_{j}\right) \text { and } m_{j}=E\left(\mu_{j}\right) .
$$

Lemma 3.

$$
\sum m_{j}=(N / \sqrt{3})+O\left\{N^{11 / 13}\left(\log n^{3 / 13}\right\}\right.
$$

Proof. This is lemma 16 of [3]. 


\section{PROOF OF THE THEOREM.}

First we consider the interval $(\varepsilon, \pi-\varepsilon)$. We have

$$
\begin{aligned}
\operatorname{Var} N(\varepsilon, \pi-\varepsilon) & \leq 4 \mathrm{E}\left\{\sum_{j}\left(N_{j}-\mu_{j}\right)\right\}^{2} \\
& +4 E\left\{\sum_{j}\left(\mu_{j}-m_{j}\right)\right\}^{2}+4 E\left\{\sum_{j}\left(m_{j}-\alpha_{j}\right)\right\}^{2} .
\end{aligned}
$$

From (2.1) and lemma 2 we have

$$
\begin{aligned}
E\left[\sum_{j}\left(N_{j}-\mu_{j}\right)^{2}\right] & \leq E\left[\sum_{j} N_{j}^{*}\right]^{2}<s E\left[\sum_{j=1}^{s}\left(N_{j}^{*}\right)^{2}\right] \\
& \leq \frac{\pi}{\delta} \sum_{j=1}^{s} E\left(N_{j}^{*}\right)<A \pi \mathrm{sn}^{3} \delta^{2} \log n .
\end{aligned}
$$

So far $\delta=o(1 / \mathrm{n})$ has been an arbitrary constant; now since the total number of $\delta$ intervals is $(\pi-2 \varepsilon) / \delta$, we choose $\delta$ such that

$$
(\pi-2 \varepsilon) / \delta=n^{15 / 13}(\log n)^{-3 / 13} .
$$

So from (3.2) we have

$$
\text { (3.3) } E \sum_{j}\left\{\left(N_{j}-\mu_{j}\right)\right\}^{2}<\operatorname{An}^{24 / 13}(\log n)^{16 / 13} \text {. }
$$

Also from lemma 3 and the fact that 


$$
\sum_{j} \alpha_{j}=n / \sqrt{3}+O\left\{n^{11 / 13}(\log n)^{3 / 13}\right\}
$$

we have

$$
\text { (3.4) } \begin{aligned}
E\left\{\sum_{j}\left(m_{j}-\alpha_{j}\right)\right\}^{2} & =E\left[n / \sqrt{3}+O\left\{n^{11 / 13}(\log n)^{3 / 13}\right\}-n / \sqrt{3}\right. \\
& \left.+O\left\{n^{11 / 13}(\log n)^{3 / 13}\right\}\right]^{2}=O\left\{n^{22 / 13}(\log n)^{6 / 13}\right\} .
\end{aligned}
$$

Hence from (3.1), (3.2), (3.3) and since from [3, page 81]

$$
E\left[\sum_{j}\left(\mu_{j}-m_{j}\right)\right]^{2}=O\left\{n^{22 / 13}(\log n)^{6 / 13}\right\}
$$

we have

$$
\text { (3.5) } \operatorname{Var} \mathrm{N}(\varepsilon, \pi-\varepsilon)=\mathrm{O}\left(\mathrm{n}^{24 / 13}(\log \mathrm{n})^{16 / 13}\right) \text {. }
$$

To find the variance in the interval $(-\varepsilon, \varepsilon)$ let $\eta(r)=\eta(r, \omega)$ be the number of zeros of $T(\theta)$ in the circle $|z| \leq r$. From [3, page 83] we know that outside an exceptional set of measure at most $\exp \left(-\mathrm{n}^{2} / 2\right)+(2 \pi)^{1 / 2} / \mathrm{n}$

$$
\eta(\varepsilon) \leq 1+(2 \log n+2 n \varepsilon) / \log 2 .
$$

Since the number of real roots in the segment of the real axis joining points $\pm \varepsilon$ does not exceed the number in the circle $|z| \leq \varepsilon$, we can obtain

$$
\text { (3.6) } N(-\varepsilon, \varepsilon)=O\left\{n^{7 / 13}(\log n)^{-4 / 13}\right\}
$$

except for sample functions in an $\omega$ - set of measure not exceeding $\exp \left(-\mathrm{n}^{2} / 2\right)+$ 
$(2 \pi)^{1 / 2} / n$. Now let $d$ be any integer of $O\left\{n^{7 / 13}(\log n)^{-4 / 13}\right\}$, then since the trigonometric polynomial has at most $2 \mathrm{n}$ zeros in $(0,2 \pi)$ from (3.6) we have

$$
\text { (3.7) } \begin{aligned}
\operatorname{Var} N(-\varepsilon, \varepsilon) & \leq \sum_{i=0}^{2 n} i^{2} \operatorname{Prob}(N=i) \\
& =\sum_{i \leq d} i^{2} \operatorname{Prob}(N=i)+\sum_{i>d}^{2 n} i^{2} \operatorname{Prob}(N=i) \\
& <B n^{23 / 13} \operatorname{Prob}\left\{N<C n^{7 / 13}(\log n)^{-4 / 13}\right\} \\
& +4 n^{2} \operatorname{Prob}\left\{N>C^{\prime} n^{7 / 13}(\log n)^{-4 / 13}\right\} \\
& <D n^{23 / 13}+4 n^{2}\left\{\exp \left(-n^{2} / 2\right)+(\sqrt{2 / \pi}) / n\right\} \\
& =O\left(n^{23 / 13}\right),
\end{aligned}
$$

where B, C, C' and D are constants. Finally from (3.5) and (3.7) we have proof of the theorem.

Remark. Although in this paper we assumed that the coefficients $g_{i}(\omega), i=1,2$, ..., $\mathrm{n}$ are independent with means zero and variance one, we can show that our theorem for the case of dependent coefficients with mean zero or non-zero (finite or infinite) and any finite variance would remain valid. However a subsequent study could be directed to reduce the upper bound obtained in our theorem, or further, to establish an asymptotic formula for the variance. 


\section{REFERENCES}

[1] Bharucha-Reid, A., Sambandham, M., Random Polynomials, Academic Press, N.Y. (1986).

[2] Cramer, H., Leadbetter, M.R., Stationary and Related Stochastic Processes, Wiley, N.Y. (1967).

[3] Dunnage, J.E.A., The number of real zeros of a random trigonometric polynomial. Proc. London Math. Soc. 16: 53-84 (1966).

[4] Farahmand, K., On the number of real zeros of a random trigonometric polynomial: coefficients with non-zero infinite mean. Stochastic Analysis and Appl. 5(4): 379-386 (1987).

[5] Qualls, C., On the number of zeros of a stationary Gaussian random trigonometric polynomial. J. London Math. Soc. 2(2): 216-220 (1970).

[6] Renganathan, N., Sambandham, M., On the average number of real zeros of a random trigonometric polynomial with dependent coefficients. II. Indian J. Pure and Appl. Math. 15(9): 951-956 (1984).

[7] Sambandham, M., On random trigonometric polynomials. Indian J. Pure and Appl. Math. 7: 841-849 (1976).

[8] Sambandham, M., On the number of real zeros of a random trigonometric polynomial. Trans. of Amer. Math. Soc. 238: $57-70$ (1978).

[9] Sambandham, M., Renganathan, N., On the number of real zeros of a random trigonometric polynomial: coefficient with non-zero mean. J. of Indian Math. Soc. 45: 193-203 (1981). 


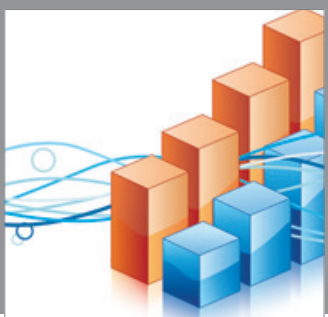

Advances in

Operations Research

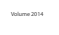

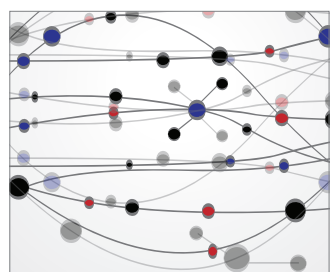

\section{The Scientific} World Journal
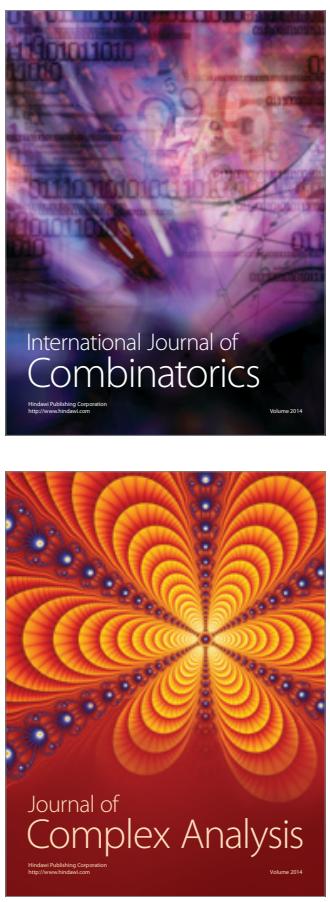

International Journal of

Mathematics and

Mathematical

Sciences
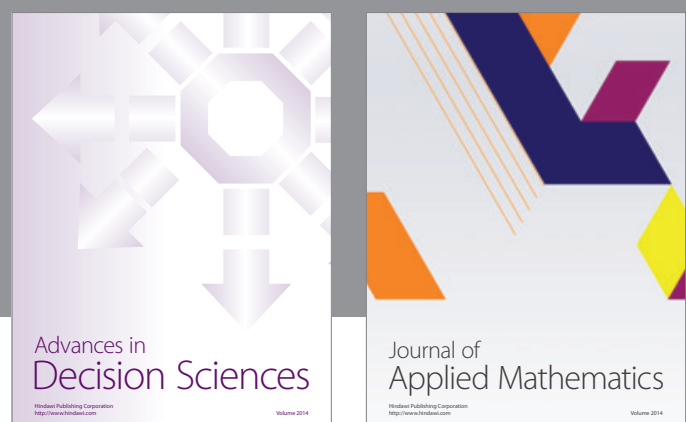

Journal of

Applied Mathematics
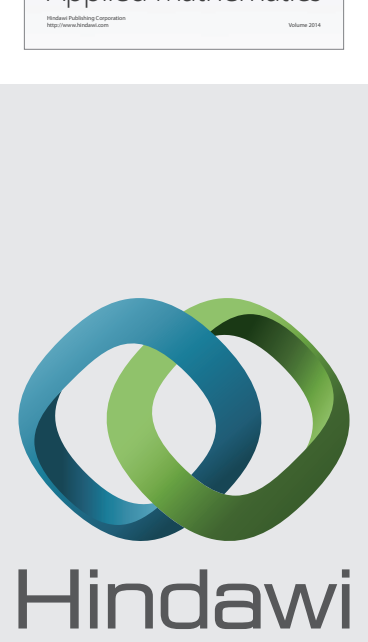

Submit your manuscripts at http://www.hindawi.com
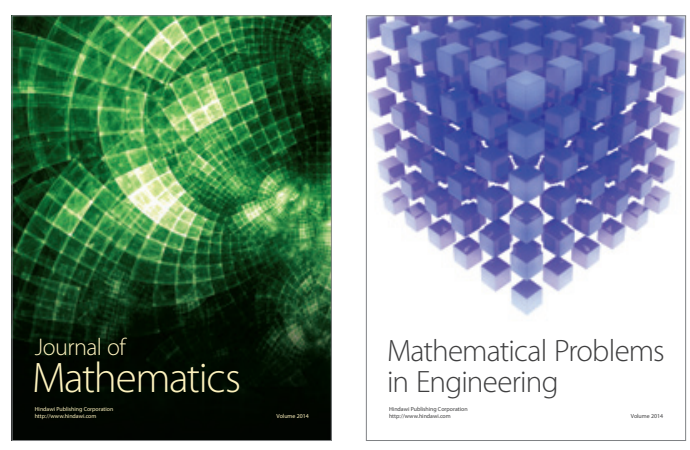

Mathematical Problems in Engineering
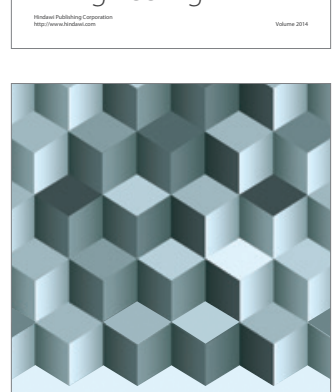

Journal of

Function Spaces
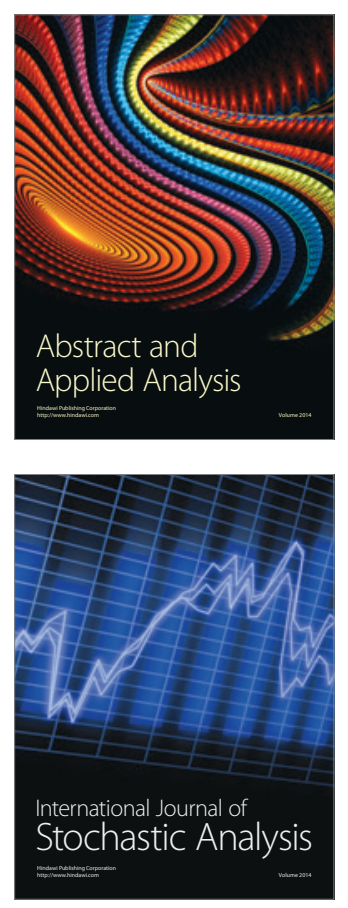

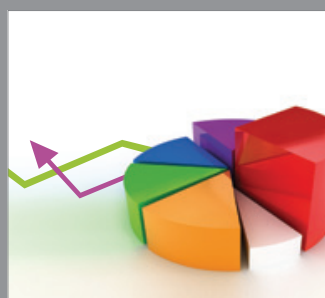

ournal of

Probability and Statistics

Promensencen
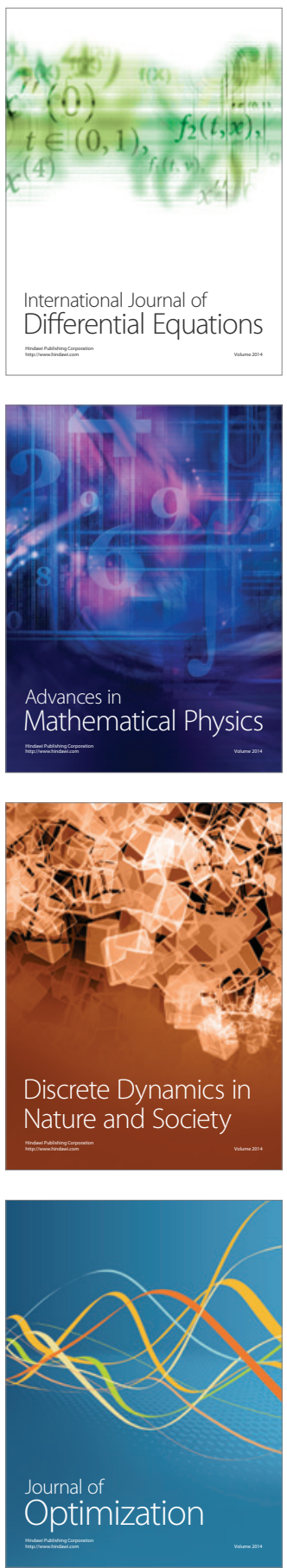